\title{
VORWORT ZUR DEUTSCHEN AUSGABE
}

Wenn ich der an mich ergangenen Aufforderung des Herrn Dr. AGTE zur Durchführung der deutschen Herausgabe des vorliegenden Buches durch den Akademie-Verlag Folge leistete, so geschah dies einmal deshalb, weil meiner Ansicht nach im deutschen Schrifttum kein ähnliches, aus so umfassender praktischer Sachkenntnis heraus geschriebenes Werk vorliegt und weil es für mich einen besonderen Reiz besaß, die experimentellen Arbeiten an Wolfram und Molybdän, die mit bescheidenen eigenen theoretischen und praktischen Arbeiten konform gehen, dem deutschsprachigen Interessenkreis in dieser Form vorzulegen. Dabei gedenke ich gern und dankbar der freundschaftlichen Einstellung der Herren Dr. AGTE und Dr. VACEK zu meiner Aufgabe, die mir Änderungen nach Belieben anzubringen gestatteten. Wenn ich von diesem vertrauensvollen Anerbieten nur in allerbeschränktestem Ausmaße Gebrauch gemacht habe, so geschah das nach reiflicher Überlegung vor allem deshalb, weil meiner Ansicht nach eine an sich leicht mögliche Heranziehung weiterer Literatur die Einheitlichkeit des Buches sprengen würde. Eine erhöhte Anzahl von Einzelfakten würde keinesfalls das grundsätzliche Bild dieses Buches entscheidend beeinflussen oder zur weiteren Klärung der Eigenschaften und Bearbeitungsvorgänge für Wolfram und Molybdän beitragen.

Ich glaube, daß die deutsche Ausgabe des Buches in der Auswahl des zur Stützung seiner Argumentation herangezogenen Schrifttums für den Interessentenkreis bedeutungsvoll sein sollte. Es zeigt, welchen erheblichen Anteil der namentlich besonders hervorzuhebende Kreis von Pulvermetallurgen an der erfolgreichen Bearbeitung dieses wissenschaftlichen und technischen Gebietes mit dem heutigen Stand hat.

Besonders wertvolle Unterstützung wurde mir durch Frl. JUTTA RIEL zuteil, welche bei der Übertragung in den deutschen Text einen wertvollen Beitrag leistete.

Gleichzeitig spreche ich hiermit meinen Dank allen Mitarbeitern aus, welche sich beim Korrekturlesen eingesetzt haben. 
